\title{
Las neurociencias en educación. ¿Un reto para los profesores?
}

Hernández Méndez, Griselda

Universidad Veracruzana, México

griseldahm2001@yahoo.com.mx

Ramírez Posadas, Maricruz

Universidad de Quintana Roo, México

mramirez@uqroo.edu.mx
Resumen - El presente ensayo plantea como preguntas ¿Los profesores están preparados para implementar en el aula la neurociencia? ¿Se les ha formado eficientemente para ello? ¿Los profesores perciben la neurociencia como un aporte a la enseñanza y al aprendizaje o una exigencia por parte de las autoridades encargadas de cristalizar las reformas educativas? El objetivo es dilucidar esa y otras interrogantes que asaltan a lo largo del ensayo, a partir de la tesis de que muchos profesores, al percibir más la exigencia o mandato de usar la neurociencia, se resisten a comprenden lo que es $y$, por ende, ignoran los beneficios que tiene en el logro de aprendizajes significativos, emotivos, duraderos y vivenciales.

Palabras claves: neurociencias; cerebro; aprendizaje; reforma educativa; México;

\author{
Hernández Méndez, Edith \\ Universidad de Quintana Roo, México \\ hernandez147@gmail.com
}

\begin{abstract}
This essay raises the central question: is neuroscience a contribution to teaching and learning or a requirement of the authorities responsible for crystallizing educational reforms? The objective is to elucidate this and other questions that assault throughout the essay, based on the thesis that many professors, perceiving more the requirement or mandate to use neuroscience, resist to understand what it is and, therefore, ignore the benefits that it has for the achievement of significant, emotional, lasting and experiential learning.
\end{abstract}

Keywords: neurosciences; brain; learning; educational reforms; Mexi$\mathrm{co}$;
Interconectando Saberes, 2019

ISSN: 2448-8704
Fecha de Recepción: 23 de agosto de 2019

Fecha de Aceptación: 14 de octubre de 2019

Fecha de Publicación: 31 de octubre de 2019 


\section{INTRODUCCIÓN}

La pedagogía, como disciplina que se encarga de la educación, y la didáctica, de la enseñanza, tienen el cometido de buscar formas imponderables de lograr un mejor aprendizaje; para lo cual, diversas teorías del aprendizaje proporcionan aportes sustanciales. Gracias al conductismo, se logró reconocer el papel del conexionismo, el uso de recompensas y castigos, los reforzamientos, la instrucción programada, entre otros aspectos; que sí bien podemos discutir su aplicabilidad y resultados, su incidencia en el aprendizaje fue notable y de gran aporte para fundamentar y mejorar la enseñanza tradicional. Posteriormente, la teoría cognitiva trajo consigo aportes para comprender cómo aprenden los estudiantes, cómo se acercan al conocimiento e incrementan esquemas de saberes a través de los procesos de asimilación, acomodación, adaptación y equilibrio. A diferencia del conductismo, para el cognoscitivismo, cada sujeto o individuo aprende de diferente manera, es decir, sus procesos de aprendizaje son distintos. Así, bajo esas ideas, el Humanismo o más concretamente, el enfoque centrado en el estudiante, asume la diferencia existente entre los estudiantes, centrándose en las emociones, sentimientos y actitudes.

Como colofón, podríamos traer a colación otras teorías necesarias, verbigracia, el psicoanálisis, la teoría social de Bandura, Erikson y más, puesto que todas ellas permiten analizar al alumno como un ser completo, integral y, a su vez complejo, pues para aprender moviliza no solo el cerebro, también está en juego su corazón, su cuerpo o físico, su biología e incuso su sexualidad, a ello se agrega que es ser social, relacional y sus emociones forman parte del aprender.

Se observa sencillo, pero aplicar las teorías con pertinencia y efectividad no es nada fácil y más si quienes imparten clases no fueron formados pedagógicamente. La pedagogía se transforma para responder a las nuevas exigencias educativas y a los estudiantes, que son distintos a los de antaño.

El siglo $X X I$ requiere de agentes pensantes -tanto maestros como alumnos-, innovadores, transformadores, competentes no solamente 
en el manejo de teorías, sino además en habilidades y destrezas para responder a los problemas del mundo y del campo laboral. De allí que cada Reforma Educativa exija que los profesores se formen para transformar el aula con lo más nuevo. En ese orden de ideas, la neurociencia, se perfila como lo más innovador en el terreno educativo. Sin embargo, ¿Los profesores están preparados para implementar en el aula la neurociencia? ¿Se les ha formado eficientemente para ello? ¿Los profesores perciben la neurociencia como un aporte a la enseñanza y al aprendizaje o una exigencia por parte de las autoridades encargadas de cristalizar las reformas educativas?

El presente ensayo tiene como objetivo dilucidar dichas interrogantes, a partir de la tesis de que muchos profesores, al percibir más la exigencia o mandato de usar la neurociencia, se resisten a comprender lo qué es la neurociencia y, por ende, ignoran los beneficios que tiene para el logro de aprendizajes significativos, emotivos, duraderos y vivenciales.
DESARROLLO

La reforma educativa.

Recomendaciones exógenas

En el documento Educación para la democracia y el desarrollo de México (2018), el Instituto Nacional para la Evaluación de la Educación (INEE), recomienda que la formación inicial de los docentes se actualice de acuerdo con los avances científicos de diversas disciplinas, entre ellas la neurociencia. En ese mismo tenor, fue escrito el Modelo Educativos para la educación obligatoria, que enfatiza la formación científica de los docentes del siglo XXI (SEP, 2018).

Por supuesto, esta recomendación se puede leer en diversos documentos de gobierno, que centran su atención en los profesores para que sean ellos los que resuelvan los males educativos: reprobación, deserción, ausentismo, bajo aprovechamiento escolar, rezago... Naturalmente, los profesores por sí solos se ven imposibilitados de resarcir todos esos males y no logran comprender las propuestas innovadoras o de reforma, ya que apenas medio están aprendiendo una, y ya viene otra. 
Hernández (2011) señala que las incertidumbres de la mayoría de los maestros, al interpretar la reforma y sus implicaciones, hacen notar que se trata de una propuesta exógena, recomendada de arriba a abajo, sin pleno conocimiento de lo que pasa en las aulas con estudiantes de carne y hueso que viven cada uno una problemática personal-familiar. Aunado a que lo encargados de las reformas no siempre cuentan con experiencias en las aulas, los cursos que se ofrecen para formar a los profesores no han sido muy eficientes; algunos son totalmente teóricos y otros, tan prácticos que caen en el reduccionismo instrumental, por lo cual inhiben la reflexión y la creatividad.

Para el caso de la neurociencia, de llano su nombre se escucha tan técnico que genera desidia, aprensión, o evasión de muchos de los profesores, y es que, en efecto, los libros y los cursos están tan cargados de tecnicismos que el maestro termina por dejar de lado intentar entender, y obviamente, no implementa.
En el instrumento Perfil, Parámetros e Indicadores para Docentes y Técnicos Docentes, utilizado en el marco del concurso de oposición para el ingreso a la Educación Básica, se percibe la exigencia de la SEP hacia la implementación de las innovaciones educativas, y concretamente de las neurociencias, sosteniendo que un docente que conoce a sus alumnos sabe cómo aprenden y lo que deben aprender".
¿Qué es la neurociencia o neurociencias y cuál es su utilidad para el aprendizaje?

Las neurociencias son disciplinas científicas que se encargan de estudiar al sistema nervioso y permiten entender, con ayuda de la tecnología, el funcionamiento del cerebro. Su uso actual corresponde a la necesidad de integrar las contribuciones de las diversas áreas de la investigación científica y de las ciencias clínicas para la comprensión del funcionamiento del sistema nervioso (Rocha-Miranda, 2001). Las neurociencias cobijan un área del conocimiento que se encarga del estudio del sistema nervioso desde el funcionamiento neuronal hasta el comportamiento. Así, el propósito principal de las neuro- 
ciencias es explicar cómo el encéfalo produce la perceptible individualidad de la acción humana, cómo actúan millones de células nerviosas individuales en el encéfalo para producir la conducta y cómo, a su vez, estas células están influidas por el medio ambiente, incluyendo la conducta de otros individuos (Kandell, Schwartz \& Jessell, 1997).

Veamos cómo funciona el cerebro en especial para que se dé el aprendizaje:

El ser humano recibe a través del sistema nervioso central información tanto interna como externa, que es procesada por el cerebro para poder dar respuesta. En el cerebro, hay miles de millones de células nerviosas llamadas neuronas y gliales, que se encargan de recibir y enviar información a otras células y órganos. Cada neurona está compuesta de un cuerpo celular, miles de pequeñas dentritas y un axón (Schunk, 2012, p.67).

Por lo tanto, cada neurona recibe a través de las dentritas los impulsos nerviosos de otras células, y el axón tiene la función contraria, transmitir el impulso nervioso a otra célula u órgano. El axón tiene una prolongación cubierta por mielina -sustancia que facilita la transmisión de la información eléctrica-, en cuyo extremo reúne unos químicos llamados neurotransmisores, liberados fuera de la neurona hacia las dentritas de otra neurona, unión que recibe el nombre de sinapsis.

El espacio que existe entre el axón de una neurona y la dentrita de otra neurona se le llama espacio sináptico. El proceso que empieza enviando un impulso eléctrico y después químico de neurona a neurona continua rápidamente con las demás formando una embrollada estructura de redes neuronales que las comunica entre sí. Obviamente este proceso se relaciona con el aprendizaje.

Para el psicólogo Schunk (2012, p.68) "el aprendizaje es el proceso de construir y modificar conexiones y redes nerviosas", esto es, tanto las experiencias diarias, como las educativas modifican y enriquecen las conexiones nerviosas haciéndolas únicas e individuales. De allí que sea de gran relevancia las situaciones de aprendizaje que se generen en el aula. 
A partir de las bases de la neurociencia, podemos inferir la relación del cerebro con el aprendizaje, a través de los siguientes puntos:

a) Una de las características del cerebro es su plasticidad, crea y modifica sus redes nerviosas continuamente a través de las variadas experiencias, vivencias y estímulos. El cerebro tiene la capacidad de aprender, reaprender y desaprender, adaptarse y buscar el equilibro. Esa consideración la tiene presente el cognoscitivismo, justo cuando Piaget habla de asimilación, acomodación, adaptación y equilibrio y es, para los profesores una invitación a ampliar esquemas de pensamiento en sus alumnos partiendo de saberes previos.

b) Cada cerebro es individual y único, como las huellas dactilares, puesto que, aunque existen patrones de funcionamiento cerebral que son comunes entre los seres humanos, cada cerebro arma y organiza su propia red neuronal. Ello significa que cada alumno aprende de diferente manera al organizar su red de neuronas y comportarse de distintas formas en las actividades que coordina el profesor o la profesora. Por eso, entre más diversas actividades se desarrollen en el aula, es mejor para que participen todos los alumnos con sus gamas de respuestas. En ciertas actividades, para algunos las experiencias serán más significativas que para otros, de allí la necesidad de la diversidad de estrategias de enseñanza.

c) El cerebro es dinámico, siempre está moviéndose y cambiando a partir de lo que experimenta. De acuerdo con Tokuhama (2013), "cada sabor, cada olor, cada visión y contacto que usted experimenta y cada sentimiento o pensamiento que usted tiene altera la forma física del cerebro" ( $p$. 20). No obstante, para que los cambios sean permanentes, se requiere del esfuerzo, de la repetición, práctica e incluso de experimentar para que las conexiones que hace el cerebro no se pierdan. Esa repetición no significa que el dictado reiterativo o la elaboración de planas sean óptimos recursos didácticos, no.

Este señalamiento deja ver que para que el alumno logre adherir el conocimiento y habilidades, se requiere de la práctica, el ejercicio, la repetición y del vivir de cerca el aprendizaje o lo que bien decía Dewey 1967): "aprender en la vida y para la vida”. 
d) El cerebro necesita de la experiencia corporal, porque está diseñado para aprender de la información que obtiene de los sentidos y del movimiento corporal. Por eso, Campos (2010) afirma que los ejercicios y el movimiento desarrollan una mejor oxigenación al cerebro, mejoran habilidades cognitivas, capacidades mentales, sociales y emocionales. Sobre todo, para los niños, el movimiento es esencial, mantenerlos pasivos, sentados en su mesa-banco es un atentado contra su propia naturaleza. Son activos, jamás pasivos $y$, si el cerebro necesita del movimiento para su oxigenación, nada mejor que incorporar el juego en el aprendizaje, o las técnicas de enseñanza que permiten moverse a los jóvenes de licenciatura, ¿quién dijo que por no ser niños no quieren o requieren moverse?

e) El cerebro demanda de conocimientos previos para el aprendizaje, pues vincular la información nueva con la anterior hace que el aprendizaje se dé y que las estructuras ya presentes adquieran sentido. El estudiante no es una tabula rasa; es decir, su mente no está en blanco, ya que hay en esta un cúmulo de conocimientos previos, experiencias, sa- beres cotidianos y teóricos... La tarea del profesor es engancharlos con los conocimientos a aprenderse y, dicho enganche no debía ser automático.

f) El cerebro tiene reacciones químicas, que varían en función de las experiencias del ambiente. El profesor, con sus actitudes y el ambiente que genera en el aula, puede activar o remplazar los componentes químicos del cerebro de sus alumnos. Así, por ejemplo, un ambiente áulico de armonía y confianza contribuye a que los neurotransmisores del cerebro liberen sustancias como la endorfina, la dopamina y la serotonina, que se verán reflejados en alumnos que pueden concentrarse en las tareas escolares y, por tanto, estar dispuestos y motivados para aprender. Ese ambiente los relaja y los optimiza para aprender. Sin embargo, un ambiente que genera altos niveles de estrés o de desconfianza, libera adrenalina y cortisol, lo que puede provocar en los estudiantes ansiedad, temor o inseguridad, y por ende, impedir que se concentren y aprendan. Si ya de por sí hay materias, como las matemáticas que son difíciles de aprenderse por el nivel de abstracción, y a eso 
se agregan las actitudes de despotismo o tiranía del profesor, ya podemos imaginar la serie de sustancias que expide el cerebro.

g) El cerebro se vitaliza, como si rejuveneciera, gracias a lo que le provoca deleite o felicidad, como el juego, el arte y la música, ya que "genera una reserva cognitiva..." (Garduño, 2019 , p.2), es decir, compensa el deterioro cerebral ocasionado por la edad o por alguna enfermedad, mejorando el rendimiento y no haciendo visible el daño cerebral en la vida diaria, pues de cierto es que mueren muchas neuronas. Por eso, muchos de los profesores con vocación, amantes de su profesión, se mantienen joviales al estar implicados con sus tareas y disfrutar de dar clases y convivir ya con niños, adolescentes o jóvenes, y más si se deleitan con el arte, la música o de otra actividad que le provoque disfrute.

h) El cerebro es selectivo, aprende solo lo que es importante y de utilidad. Hay tanto que aprender del mundo, de la vida, del existir, de lo inmediato y mediato, etc., por eso el humano nunca acaba de aprender. Nuestro mundo está rodeado de constantes estímulos sensoriales, y eso lo sabe el cerebro; por eso es selectivo, filtra aquello que no le impida concentrase en lo que le interesa adherir, evade lo que considera accesorio o de poca utilidad. Por eso a los estudiantes se les dificulta aprender aquello que no le ven utilidad o les es complicado; su cerebro está ocupado aprendiendo aquello que sí es interesante como las múltiples informaciones de las redes sociales, series o películas...para ellos eso es estimulante y no las clases parcas y sin vida de las aulas.

Si el cerebro solo se queda con lo más estimulante, es necesario que se enseñe lo verdaderamente útil y, sobretodo, que el maestro sea en sí mismo un motivador, que esté convencido que lo que enseña sirve y busque formas de hacer el aprendizaje placentero. Al ser selectivo el cerebro, se aburre ante lo que no genera interés. Hecho que deberá tomar en cuenta el profesor si desea que sus estudiantes estén atentos y que retengan la información.

i) El cerebro demanda de un derrotero de aprendizaje; es decir, necesita una ruta, un diseño planificado para que se constituya una estructura, se asimile el saber, acomode y 
reacomoden esquemas de pensamiento, se repase y quede en la memoria con la organización de la información. El ejercicio, el estímulo, la reafirmación son indispensables para asir el saber. Esto exige al profesor la planificación de actividades didácticas favorecedoras para aprender con complacencia y no con animadversión (Hernández, 2009 en Landgrave, Flores, Montfort y Fabre).

Mucho se necesita de conocer a las neurociencias, pues a veces se generan creencias erradas como la premisa de que los hemisferios del cerebro funcionan de manera aislada realizando tareas específicas. En realidad, está probado que "ambos hemisferios están unidos por fibras y trabajan como un complejo diseño de sistemas integrados" (Schunk, 2012, p. 33); es decir, desarrollan un trabajo conjunto en el proceso de aprendizaje. Los profesores asisten a cursos donde se muestra esa división del cerebro o de "estilo" y se les exhorta a la atención de dicha separación, cuando es un error y un trabajo titánico sin sentido.
Los profesores, en su mayoría, se decepcionan al ver que sus esfuerzos son estériles porque los alumnos no aprenden más, como se les hace creer en esos cursos, más técnicos que de reflexión y análisis. Por eso terminan rechazando las indicaciones de las reformas educativas, pues pareciera que quien las propone desconociera la vida en las aulas.

Sin embargo, como pudimos darnos cuenta, llevar al aula los aportes de las neurociencias es un reto que vale la pena.

\section{CONCLUSIONES Y}

\section{REFLEXIONES}

A partir de los puntos revisados, podemos concluir que las neurociencias alcanzan aportes de riqueza excepcional para el logro del aprendizaje. No es necesario tanto tecnicismo, basta con que los profesores conozcan lo básico y modifiquen algunas de sus formas de enseñar con el afán de hacer más estimulantes y enriquecedores los aprendizajes. 
Queda claro que cada alumno aprende a su modo y en ritmos distintos, debido a factores genéticos, el tamaño del cerebro varía, así como la forma en que se entretejen las redes nerviosas, por eso algunos aprenden más rápido y otros no, algunos tienen habilidades que a otros les faltan. Sin embargo, ha de resaltarse que las habilidades pueden perderse si no se desarroIlan o enaltecen. "Las personas nacen con diferentes habilidades, las cuales pueden desarrollar o perder dependiendo de los estímulos o la falta de ellos" (Tokuhama, 2013, p.19).

Por ello, es fundamental que el profesor se mire como un verdadero estimulador, de lo que haga en el aula, dependerá que el estudiante aprenda. Por eso es tan desgastante el enseñar potencializando las habilidades de los alumnos, pero también muy gratificante cuando el alumno demuestra ser hábil gracias al apoyo de su profesor.

Es necesario que todo profesor, independientemente del nivel educativo en el que se desempeñe, se pregunté sobre qué puede hacer para lograr aprendizajes significativos, emotivos y permanentes; ello lo llevará a recurrir a la neurodidáctica, indispensable para el desarrollo cerebral.

El ambiente áulico debe ser estimulante para favorecer el desarrollo cerebral y las conexiones nerviosas. Un profesor facilitador cuida del ambiente humano de confianza porque sabe que los predispone a estar tranquilos, relajados y más abiertos a aprender. Desde casa, los padres deben ser cuidadosos con sus hijos, hablarles con cariño, acercarlos a la música, la lectura, y los idiomas por deleite, porque ello traerá consigo estudiantes más prestos al desarrollo de capacidades.

Por tanto, la respuesta a nuestras preguntas iniciales son fácil de responder, las neurociencias son un gran aporte para la enseñanza y el aprendizaje, pero las autoridades encargadas de proponerlas como innovaciones educativas, deberían cuidar las formas en que se hacen llegar al profesorado. No como imposiciones exógenas sino como verdaderos retos para la mejora en los aprendizajes. 


\section{BIBLIOGRAFÍA}

Campos, A. L. (junio, 2010). Neuroeducación: uniendo las neurociencias y la educación en la búsqueda del desarrollo humano. La educ@ción.Digital Magazine, 143. Recuperado de http://www.educoea.org/portal/La_Ed ucacion_Digital/laeducacion_143/arti cles/neuroeducacion.pdf

Dewey, J. (1967). Mi credo pedagógico. En: El niño y el programa escolar. Mi credo pedagógico, $6^{\mathrm{a}}$ ed., Lorenzo Luzuriaga (trad.). Buenos Aires: Losada, pp. 51-66.

Garduño, V. (febrero, 2019). Consejos de neurociencia para docentes. Recuperado de https://www.inee.edu.mx/index.php/p ublicaciones-micrositio/blog-revistared/610-blog-revista-red-home/blogrevista-red-articulos/3756-consejosde-neurociencia-para-docentes.

Hernández, G. (2011). Transformar el subsuelo antes que la superficie. Revista Educación y Humanismo, 20.

Instituto Nacional para la Evaluación de la Educación (2018). Educación para la democracia y el desarrollo de México. Recuperado de https://www.inee.edu.mx/images/stori es/2018/democracia/Educacion.pdf.

Kandell, Schwartz y Jessell (1997). Neurociencia y conducta. Traducido por Pilar Herreros de Tejada Macua, Miguel Navarro García, María José Ramos Platón y Fernando Rodríguez de Fonseca. Madrid: Prentice Hall.

Landgrave, Flores, Montfort y Fabre (2009). La complejidad en realidades diversas. México: IIESES.
Paniagua, M. N. (2013).

Neurodidáctica: una nueva forma de hacer educación. Fides et ratio. Revista de difusión cultural y científica de la Universidad la Salle Bolivia. Recuperado de http://www.ulasalle.edu.bo/fidesetrati o/images/fidesetratio/portadas/FIDE SETRATIOVol6. pdf?fbclid=IwAR0OP LQCmnKgnZlvjD2Clan0VX5U9CQeb ENXAtWyVu01E7IdfiX7v9wnhSo

Sánchez, E. (2009). Mente, cerebro y educación. Aula: Revista de Pedagogía de la Universidad de Salamanca 15. Recuperado de http://revistas.usal.es/index.php/021 4-3402/article/viewFile/6947/10425

Secretaria de Educación pública (2018) Modelo Educativo para la educación obligatoria. México: SEP.

Rocha-Miranda, (2001). Neurociencias del comportamiento. Recuperado en http://www.upbbga.edu.co/filesupb/N EUROCIENCIAS.pdf.

Schunk, D. (2013). Teorías del aprendizaje. Una perspectiva educativa. Recuperado de http://www.visam.edu.mx/archivos/_L IBRO\%206xta_Edicion_TEORIAS_D EL APRENDIZ̄AJE\%20\%20DALE\%20H\%20SCHUNK.pdf

Tokuhama, T. (2013). ¿Qué puede hacer la ciencia de Mente, Cerebro y Educación (MCE) por la enseñanza y el aprendizaje? Recuperado de https://www.usfq.edu.ec/publicacione s/para_el_aula/Documents/para_el aula_05/0010_para_el_aula_05.pdf 